\title{
MR lymphangiography in lymphedema
}

\section{Sitthiphan Limphanudom, M.D.}

Piyatida Boonsin, M.D.

From The Department of Radiology, Faculty of Medicine, Prince of Songkla University, Songkhla, Thailand Address correspondence to S.L. (e-mail: sitthiphan.l@psu.ac.th)

Lymphedema is a chronic disorder, defined as a progressive and excessive accumulation of protein-rich fluid, inflammation, adipose tissue hypertrophy, and fibrosis in the interstitial space, which results from impaired lymphatic drainage. Regardless of the etiology, it is clinically characterized by chronic swelling, localized pain, atrophic skin changes and secondary infections. However, the main devastating aspect of lymphedema is the appearance of the affected limb that causes psychological morbidity.

Lymphedema is classified as primary or secondary depending on etiology and presentation. The primary lymphedema is a form of lymphedema without an inciting factor, generally due to a congenital condition associated with pathologic development of the lymphatic vessels. It can be divided into three forms, classified by the age at the onset: congenital lymphedema, lymphedema praecox, and lymphedema tarda. Congenital lymphedema presents at birth or up to two years postnatally. Lymphedema praecox typically arises during puberty or pregnancy with the onset prior to age 35 years, which accounts for $77-94 \%$ of all cases of primary lymphedema, and lymphedema tarda presents with an onset after age 35.1,2 The secondary lymphedema occurs as a result of other conditions or treatments, usually resulting from injury of the lymphatic vessels from infection, trauma, or tumors but it may also be iatrogenic following surgical interventions or radiotherapies. In developing countries, secondary lymphedema 
is mainly caused by infections-infestations influencing lymphatic channels, primarily the result of filarial infections (Wuscheria banrofti). ${ }^{1}$ On the other hand, in developed countries, secondary lymphedema occurs most commonly after the surgical removal of lymph nodes for cancer treatment.

Despite being a common problem, it is vulnerable to misdiagnosis and has been badly reputed as an incurable disease. There are conservative and operative treatment options for lymphedema. Unfortunately, the efficacy of conservative treatment is highly variable between patients and is not curative. Nowadays, the microsurgical treatment for bypassing the obstructed segment of the lymphatic pathway into the venous system, also known as lymphovenous anastomosis (LVA) is preferred in lymphedema cases. However, a preoperative imaging technique is needed to assess the soft tissues of the entire limb, determining whether suitable lymphatic channels are present, and defining the location of the lymphatics relative to anatomic landmarks that the surgeon can use in the operation. ${ }^{3}$

There are several imaging modalities which aid in the diagnosis of lymphedema and define the location of obstruction such as lymphoscintigraphy, Near Infra-Red Fluorescence Imaging (NIRF), conventional lymphangiography, CT lymphangiography and MR lymphangiography.

Lymphoscintigraphy is a nuclear medicine study and demonstrates a slow or absent lymph flow usually in later stages of lymphedema. Technetium $99 \mathrm{~m}$ sulfur colloid is injected intradermally and the transit time to lymph node basins can be measured; however, subdermal lymphatics cannot be assessed. While radionuclide lymphoscintigraphy has been considered the primary clinical imaging modality to diagnose lymphedema, its limited temporal and spatial resolution does not allow the identification and localization of individual lymphatic channels. NIRF is a dynamic test done by using indocyanine green, allowing visualization of the superficial lymphatic flow and functioning lymphatic vessels, thus, discovering abnormalities at early stages. It can be used to stage the severity of the disease and for preoperative-intraoperative planning. Conventional lymphangiography is another entity where radio-opaque material is directly 
injected into peripheral lymph vessels, but the number of studies declined markedly since the introduction of cross-sectional imaging techniques. Therefore, levels of expertise in both performing lymphography and interpreting lymphograms are declining. This technique is rarely adopted due to the risk of damaging lymph vessels. ${ }^{4}$

MR lymphangiography (MRL) is an advanced imaging technique using 3D volumetric contrast-enhanced MR angiography with high temporal and spatial resolution, by injection of Gadolinium into the hand or foot to clarify the course of lymphatics, which can be used to evaluate both anatomy and function of the lymphatic system in a single examination. It is a quick and less invasive imaging technique for evaluation in a case of lymphedema or suspected lymphatic injury, and useful for preoperative planning of LVA microsurgery. MRL could also be useful for evaluating changes in the lymphatic circulation postoperatively or in the event of surgical complications. However, there are also limitations despite Gadolinium, which can also get into the venous system making the interpretation of lymphatic channels difficult.

The indication of MRL is for diagnosis and preoperative planning for the case of primary lymphedema. The contraindication of MRL is the same as general contraindication for MRI such as patients who have a metallic device or a metallic foreign body.

Our experience of MRL in Prince of Songkla University (PSU) hospital will be described from this point on. All the cases were referred from MRL of the lower extremities, to rule out lymphedema and evaluate the causes of lymphedema in affected limbs.

\section{Equipment and imaging techniques}

Equipment: MRL can be performed at either 1.5 tesla or 3.0 tesla. In our experience, a 3.0-tesla MRI was preferred and most of the cases were performed by a Philips Achieva $3.0 \mathrm{~T}$ dStream, with a maximum gradient strength value of $40 \mathrm{mT} / \mathrm{m}$ and a slew rate of $200 \mathrm{mT} / \mathrm{m} / \mathrm{ms}$. 
Coils: a multielement body coil is fundamental for this type of examination. Based on our purposes, we used a phased-array coil for the study of the lower extremities (SENSE XL Torso coil, Philips Medical Systems), which has a large anatomical coverage and a good signal-to-noise ratio.

Patient position: patients were placed supine and feet-first on the scanner table. The multielement coils were positioned to cover both lower extremities from both feet to the groin. Then, a two-station exam was performed.

Contrast medium: based on our purposes, the water soluble extracellular Gd-based MR contrast agent used was gadoterate meglumine (Gd-DOTA, Dotarem) with concentration of $0.5 \mathrm{mmol} / \mathrm{mL}$. A mixture of a standard dose $(0.1 \mathrm{mmol} / \mathrm{kg}$ body weight) contrast medium and $2-4 \mathrm{~mL}$ of $2 \%$ lidocaine (Xylocaine) without adrenaline for local anesthesia is injected subcutaneously/ intradermally.

Insertion of the needle: a $24-28$-Gauge thin needle is generally preferred. Ideally, the tip of the needle should gently be inserted subcutaneously into the dorsal aspect of each foot in the region of the four interdigital web spaces. The injection is limited to a maximum volume of $2 \mathrm{~mL}$ (generally $1 \mathrm{~mL}$ ) for each interdigital web space.

Imaging techniques:

- Short-tau inversion recovery (STIR) sequences in coronal view of both lower extremities before the contrast medium injection.

- 3D Spoiled gradient echo (3D GRE) of both lower extremities; dynamic at 0, 5, $15,25,35,45,55$ and 90 minutes (min), respectively.

Each sequence parameter for the 3.0-T MRL is shown as follows: 


$\begin{array}{lll}\text { Parameter } & \text { STIR } & 3 \text { D GRE } \\ \text { FOV } & 400 \times 500 \times 258 \mathrm{~mm}^{3} & 340 \times 499 \times 260 \mathrm{~mm}^{3} \\ \text { Matrix } & 525 \times 277 & 228 \times 332 \\ \text { TR }(\mathrm{ms}) & 14499 \mathrm{~ms} & 3.4 \mathrm{~ms} \\ \text { TE }(\mathrm{ms}) & 70 \mathrm{~ms} & 1.32 / 2.2 \mathrm{~ms} \\ \text { TI }(\mathrm{ms}) & 230 \mathrm{~ms} & - \\ \text { FA } & 90^{\circ} & 10^{\circ}\end{array}$

\section{Case study}

A 48-year-old female presented with progressive, having a swelling left leg for 1 month. She had been diagnosed with a left popliteal injury after a motorcycle accident for 10 months and post repair with PTFE graft.

Doppler study for both thighs showed a patent left popliteal artery graft without significant stenosis and patent left DPA and distal PTA. Deep vein ultrasound study also showed a normal venous system.

MRL of the left leg was performed on a 3.0-T machine after obtaining an informed consent. Following painting and draping of her left foot, $14 \mathrm{~mL}$ of Dotarem and 2\% Xylocain was injected subcutaneously into all the interdigital web spaces and between the first and second proximal metatarsal spaces of both feet. The injected sites were massaged for $1 \mathrm{~min}$. Imaging was performed using a STIR 3D-DRIVE and dynamic 3D GRE. The acquisition of dynamic 3D-GRE was done at $0,5,15,25,35,45,55$ and $90 \mathrm{~min}$ after injection. A dilated epifascial lymphatic system with an extensive reticular pattern were first visualized in $5 \mathrm{~min}$ (Figure 1-B) followed by $15 \mathrm{~min}$ (Figure 1-C), $35 \mathrm{~min}$ (Figure 1-D) and by 55 min, lymphatics up to the groin could be seen (Figure 1-E). There was an excellent visualization of multiple lymphatic channels on the medial aspect of the left leg and dorsum of the left foot. In addition, there was also normal contrast filling of the venous systems. No abnormal tortuous dilated lymphatic vessels or an 
obvious dermal back flow of right lower extremity was noted. The patient was kept on antibiotics after the procedure. There was no pain at the puncture site and no other complications were noted.

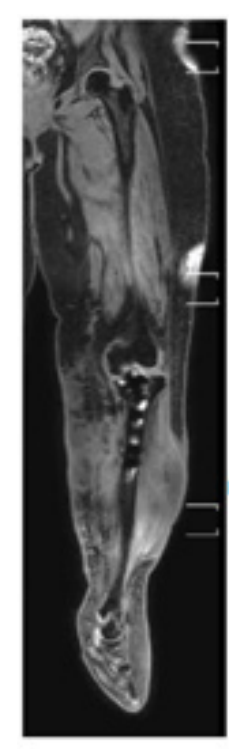

A

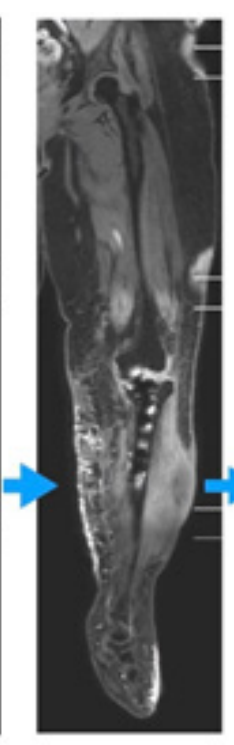

B

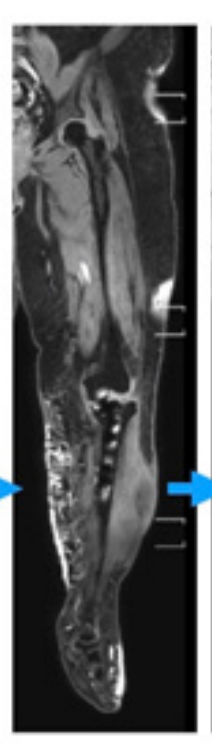

C

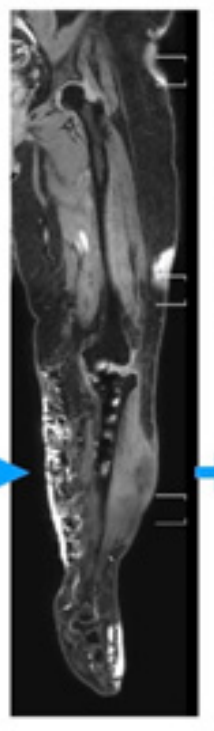

D

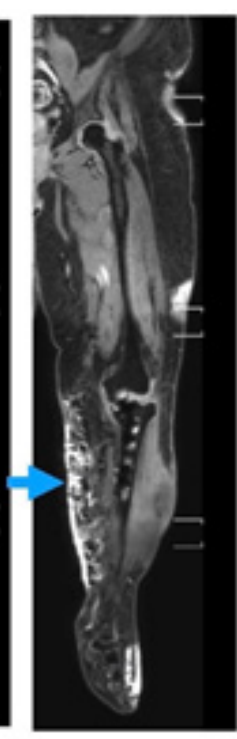

E

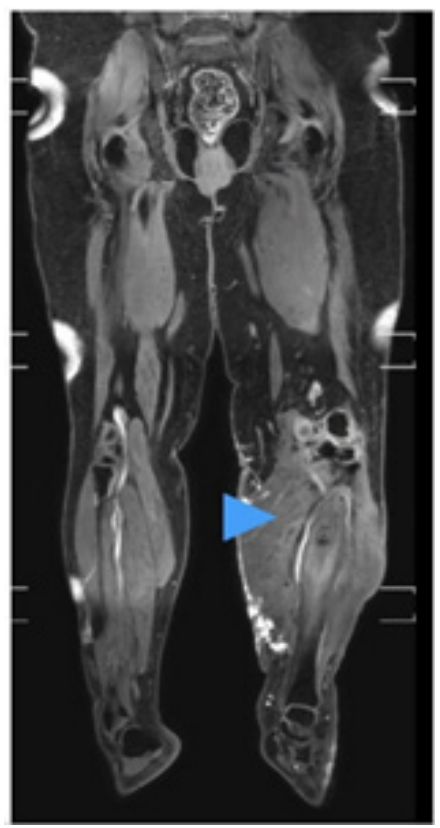

Figure 1. 3D MRI images obtained at $0 \mathrm{~min}(\mathrm{~A}), 5 \mathrm{~min}(\mathrm{~B}), 15 \mathrm{~min}(\mathrm{C}) 35 \mathrm{~min}$ (D) and 55 min (E) shows the dilated epifascial lymphatics (arrows) and a normal visualization of the veins (arrow head).

In summary, the MRI lymphangiography is a modality for diagnosing peripheral lymphedema, which can be seen as stagnation of the contrast media at the level of pathology in the affected limb, or congestion of contrast media in dilated lymphatic systems distal to obstructive point. Furthermore, the venous system can also be evaluated at the same time. MRL is considered noninvasive and more helpful for diagnosis and preoperative evaluation in a case of lymphedema for its better spatial and temporal resolution. 


\section{References}

1. Mehrara B. Clinical features and diagnosis of peripheral lymphedema [Internet]. Post TW, ed. UpToDate. Waltham, MA: UpToDate Inc. [updated 2018 Dec 17; cited 2019 Jul 14]. Available from: https://www. uptodate.com.

2. Damstra RJ, Mortimer PS. Diagnosis and therapy in children with lymphoedema. Phlebology 2008;23:276-86.

3. Baz AA, Hassan TA, Atta AT, El kholy MS. Role of contrast enhanced MRI lymphangiography in evaluation of lower extremity lymphatic vessels for patients with primary lymphedema. Egypt J Radiol Nucl Med 2018;49:776-81.

4. Kayiran O, De La Cruz C, Tane K, Soran A. Lymphedema: from diagnosis to treatment. Turk J Surg 2017;33:51-7.

5. Mitsumori LM, McDonald ES, Wilson GJ, Neligan PC, Minoshima S, Maki JH. MR lymphangiography: how i do it. J Magn Reson Imaging 2015;42:146577.

6. Mazzei FG, Gentili F, Guerrini S, Cioffi Squitieri N, Guerrieri D, Gennaro P, et al. MR Lymphangiography: a practical guide to perform It and a brief review of the literature from a technical point of view. Biomed Res Int. 2017;2017:2598358. doi: 10.1155/2017/2598358. 